\title{
Automatic Detection of Atrial Fibrillation Using Electrocardiomatrix and Convolutional Neural Network
}

\author{
Ricardo Salinas-Martínez, ${ }^{1,2}$ Johannes de Bie, ${ }^{1}$ Nicoletta Marzocchi, ${ }^{1}$ Frida Sandberg ${ }^{2}$ \\ ${ }^{1}$ Hillrom - Mortara Instrument Europe, Bologna, Italy \\ ${ }^{2}$ Department of Biomedical Engineering, Lund University, Lund, Sweden
}

\begin{abstract}
Long-term electrocardiogram (ECG) monitoring is a standard clinical routine in cryptogenic stroke survivors to assess the presence of atrial fibrillation $(A F)$. However, manual evaluation of such recordings is time consuming, in particular when brief episodes are of interest. The electrocardiomatrix (ECM) technique allows compact, two-dimensional representation of the ECG and facilitates its review. In this study, we present a convolutional neural network (CNN) approach for automatic detection of $A F$ based on ECM images. ECG segments of only 10 beats were converted into ECM images. A CNN was implemented to classify the ECMs between non-AF and AF. The CNN was trained using the MIT-BIH-NSR and the MIT-BIH-LTAF, and tested on the MIT-BIH-AF. A total of 120088 non-AF and 108088 AF ECM images were classified with accuracy of $86.95 \%$. This study suggests that a CNN allows automatic detection of AF episodes of only 10 beats when the ECG data is represented as an ECM image.
\end{abstract}

\section{Introduction}

The electrocardiogram (ECG) is a powerful clinical tool for non-invasive diagnosis of cardiovascular diseases. For this purpose, morphology and rhythm of the ECG is analyzed. This task becomes increasingly complex when the number of ECG segments is high or when the duration of the record is very long, such as in Holter monitoring [1], which is a standard clinical routine in cryptogenic stroke survivors to assess the presence of atrial fibrillation (AF) [2]. This heart rhythm disorder is the most common cardiac arrhythmia and it has been correlated with significant morbidity, mortality, and reduction of life quality [2]. Furthermore, it is associated with a two-fold increased risk of ischemic stroke, and significantly rises public health burden [2]. Nowadays, the increasing demand for even longer ECG monitoring [1] calls researchers to develop new approaches to facilitate ECG representation and interpretation. In this context, $\mathrm{Li}$ et al. presented in 2015 the electrocardiomatrix (ECM) [3]. This technique allows compact, two-dimensional representation of the ECG preserving morphology and rhythm characteristics, thus facilitating its review. Brown et al. concluded that the ECM allows superior accuracy of manually detecting AF episodes than traditional monitor analysis by noncardiologist when using telemetry data [4]. Moreover, to improve ECG interpretation and speed up decision making for treatment, computer-based detectors of AF have shown to be helpful in diagnostic procedures to avoid further lifethreatening conditions [5]. Different methodologies for automatic detection of $\mathrm{AF}$ have been proposed, some focus on the rhythm of the ECG but requires a long data buffer to provide a reliable diagnosis, while more complex approaches take into account the morphology of the signal to improve performance [6]. Additionally, with the exponential development of technology, machine learning (ML) techniques have become popular approaches among researchers for the detection of brief episodes of $\mathrm{AF}[7,8]$. Machine learning programs learn from experience with respect to some task and performance evaluation, in this way the performance of the program to do the required task increases with the experience gained [9]. In this sense, we create a big training dataset for the algorithm to extract and learn representative features for each class of interest. Convolutional neuronal networks $(\mathrm{CNN})$ is one kind ML methodology, introduced by Le Cun [10], that can be used for classification in high dimension applications [11]. In this study, we present a CNN approach for automatic detection of brief episodes of AF based on ECM images. This approach permits classification without the need for manual feature extraction or advance signal prepocessing.

\section{Data}

Three public databases available at PhysioNet [12] were used in this study; the Long-Term Atrial Fibrillation (LTAFDB), the MIT-BIH Normal Sinus Rhythm (NSRDB), and the MIT-BIH Atrial Fibrillation (AFDB) databases. The LTAFDB contains 84 two-lead long-term 
ECG recordings from patients with paroxysmal or persistent $\mathrm{AF}$ lasting from 24 to $25 \mathrm{~h}$ with a sampling frequency of $128 \mathrm{~Hz}$ [13]. The NSRDB consist of 18 two-lead longterm ECG recordings acquired with a sampling frequency of $128 \mathrm{~Hz}$ from patients without any significant arrhythmia lasting from 23 to $26 \mathrm{~h}$ [12]. Finally, the AFDB is a set of 25 two-lead long-term ECG recordings acquired with a sampling frequency of $250 \mathrm{~Hz}$ from patients with AF, mostly paroxysmal, lasting $10 \mathrm{~h} \mathrm{[14].} \mathrm{Two} \mathrm{recordings}$ from the AFDB and one recording from the LTAFDB were excluded from the study due to corrupted files.

\section{Methods}

\subsection{Generation of Electrocardiomatrix}

All recordings were first upsampled to $500 \mathrm{~Hz}$ to fulfill the requirements of The VERITAS ${ }^{\mathrm{TM}}$ algorithm from Hillrom, which was used for beat detection. Next, baseline wander was removed using a highpass filter with cutoff frequency of $0.5 \mathrm{~Hz}$. Feedforward filtering was performed to avoid phase distortion. All beats detected by the algorithm were considered, including ectopic beats. Finally, the signal was confined in amplitude by truncation to $\pm 1 \mathrm{mV}$.

The ECMs were constructed from each lead of the ECG recordings. Each signal was divided into segments containing 10 beats. A $50 \%$ overlapping window was set to create the ECMs used during training and validation while non-overlapping segments were considered to create the ECMs for testing. The same segmentation was made for the different leads. The resulting segments were then split into 10 subsegments of $3.0 \mathrm{~s}$ length, where the $i$ :th subsegment starts $0.5 \mathrm{~s}$ before the fidutial point of the $i$ :th detected beat, $1 \leq i \leq 10$. Next, the ECG subsegments were decimated in two sections: 1$)$ in the interval $[0,0.5] \mathrm{s}$ the signal was decimated to $125 \mathrm{~Hz}, 2)$ in the interval $[0.5,3] \mathrm{s}$ it was decimated to $50 \mathrm{~Hz}$. The first part is intended to keep most of the morphology of the P-wave of the $i$ :th beat whereas the second one provides information on the "local" rhythm. Finally, all subsegments were aligned vertically and converted into a matrix, this matrix will be referred to as ECM. Figures 1 and 2 illustrate the concept of creating an ECM, grayscale is used to display the ECMs with dimensions $219 \times 10$ pixels. The ECMs don't consider segments that include a transition from $\mathrm{AF}$ to non-AF or viceversa.

\subsection{Classification process}

The LTAFDB and the NSRDB were merged together, hereinafter the training database (TDB), and used for training and validation, while the AFDB was used for testing. A subset of $80 \%$ of the ECMs resulting from the TDB was used for training and the remaining $20 \%$ was used
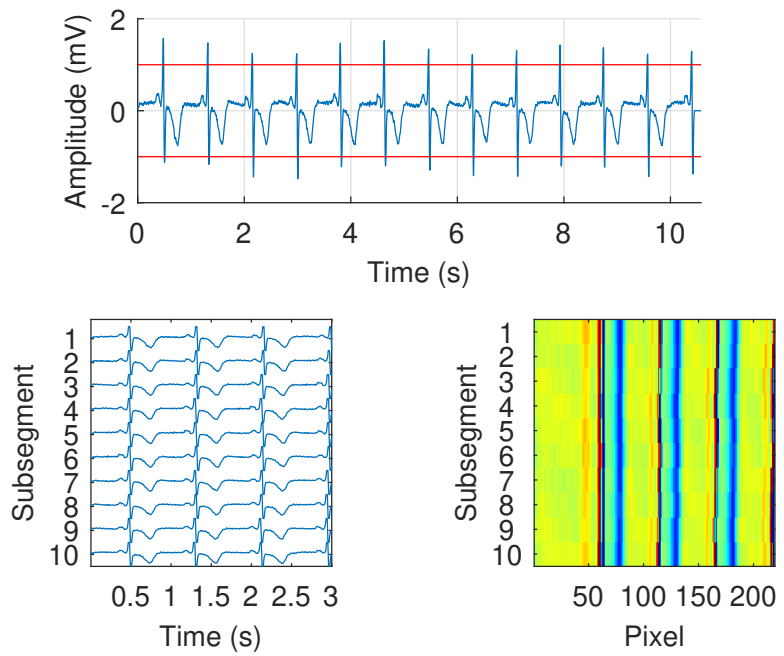

Figure 1: Generation of the ECM showing a non-AF episode. Segment taken from channel 1 of the recording 04015 in the AFDB. The segment corresponds to the time interval $[4567,4578]$ seconds.
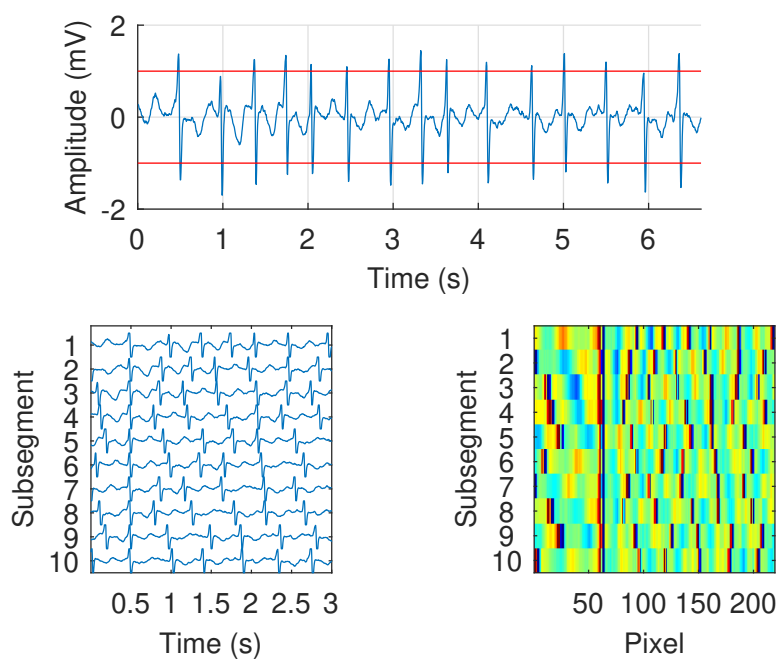

Figure 2: Generation of the ECM showing an AF episodes. Segment taken from channel 1 of the recording 04015 in the AFDB. The segment corresponds to the time interval [4385, 4391] seconds.

for validation. Three-fold cross-validation was performed with datasets selected manually, such that validation sets were always taken from different recordings than training data. The ECM classification between non-AF and AF was performed using a $\mathrm{CNN}$ composed of 3 convolutional layers, 3 batch normalization, 3 ReLu activation, 2 max pooling, 1 fully connected, 1 softmax, and 1 classification layer [15]. Figure 3 illustrates the architecture of the CNN. This architecture was selected to maintain a tradeoff between performance and computational complexity. 


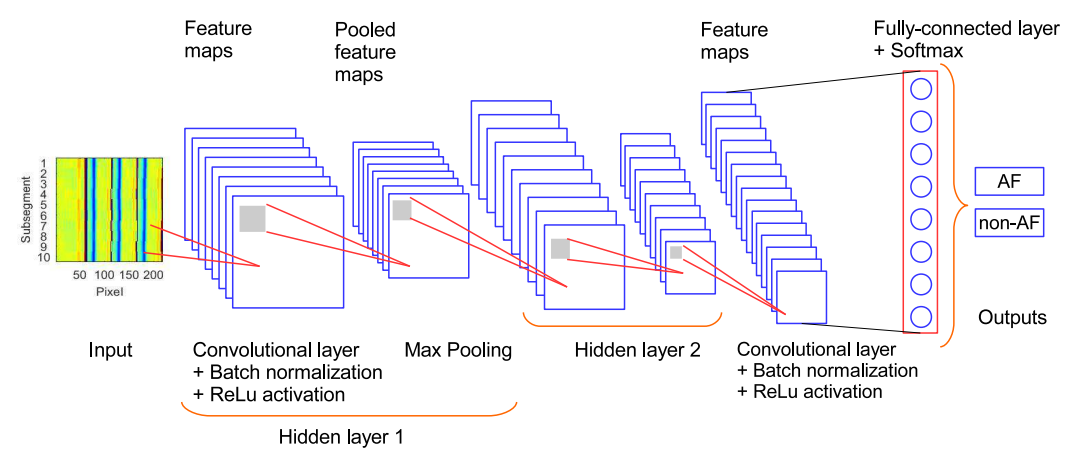

Figure 3: Architecture of the CNN used for classification.

A total of 11257 learnable parameters are contemplated. Batches of 10000 ECMs were used to speed up the training process [16], shuffling the batches on each of the 3 epochs considered. The $\mathrm{CNN}$ was trained using the function trainNetwork included in the Deep Learning Toolbox from MATLAB [17]. The stochastic gradient descent with momentum was used to solve the maximal a posteriori cost function with learning rate of 0.01 , L2 regularization factor of 0.0001 , and momentum contribution of 0.9 .

\section{Results}

Using the TDB, 2184102 and 2109744 ECM images were generated for non-AF and AF, respectively. The AFDB produced 120088 and 108088 ECM images for non-AF and AF, respectively; which were used for testing.

Accuracy of $89.83 \% \pm 3.99$ (mean \pm std) was obtained during validation, and accuracy of $85.08 \% \pm 2.33\{82.45$, $85.91,86.88\}$ was obtained during testing. Table 1 summarizes the performance of the presented approach. Next, classification was done based on the prediction made by all three networks. In this sense, the resulting labels from each network were contrasted between each other, and each ECM was placed in the class with majority of votes. The resulting accuracy was $86.95 \%$, indicating a large agreement between the trained networks.

\section{Discussion}

Once the ECG signal is presented as an ECM, a CNN can be trained for classification purposes to distinguish between non-AF and AF ECM images. This process allows automatic detection of AF episodes based on the information from only 10 beats. Additionally, the ECG signals requires minimal processing before classification in comparison with other $\mathrm{CNN}$ approaches [7,8]. Moreover, our $\mathrm{CNN}$ was trained and tested on separate databases while Xia et al [7] and He et al [8] used the same database for training and testing increasing the chances of overfitting.
Table 1: Performance achieved during validation and testing presented in terms of mean \pm standard deviation.

\begin{tabular}{|l|c|c|}
\hline & Validation & Testing \\
\hline Accuracy (\%) & $89.83 \pm 3.99$ & $85.08 \pm 2.33$ \\
\hline Precision (\%) & $91.54 \pm 3.87$ & $88.94 \pm 2.61$ \\
\hline Sensitivity (\%) & $87.94 \pm 8.48$ & $78.26 \pm 4.25$ \\
\hline Specificity (\%) & $91.17 \pm 4.29$ & $91.22 \pm 2.25$ \\
\hline
\end{tabular}
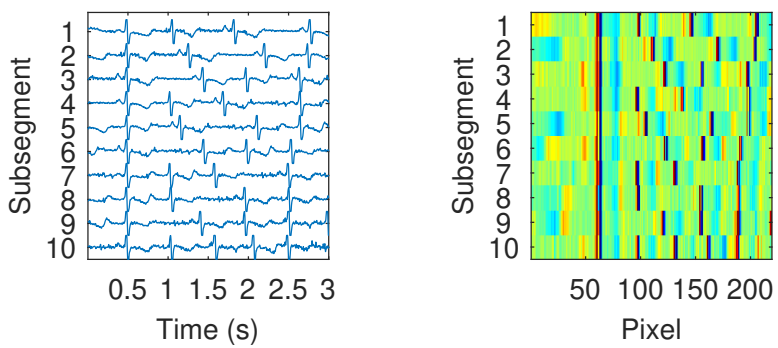

Figure 4: ECM showing a non-AF episode. False-positive misclassification probably due to the presence of bigeminy. Segment taken from channel 1 of the recording 04015 in the AFDB. The segment corresponds to the time interval $[65,75]$ seconds.
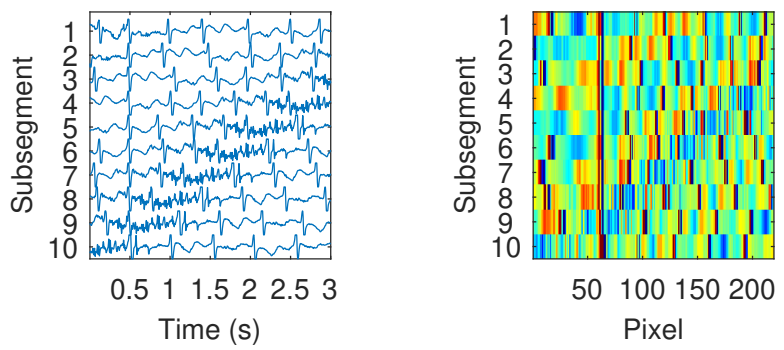

Figure 5: ECM showing an AF episode. False-negative misclassification probably due to motion artifacts. Segment taken from channel 1 of the recording 04015 in the AFDB. The segment corresponds to the time interval [428, 435] seconds. 
The classification capacity of the algorithm is affected by the presence of non-AF arrhythmias, such as bigeminy and trigeminy, and motion artifacts present in the signal. These factors may lead to false-positive when non-AF arrhythmias are present, one example of this is shown in Figure 4. On the other hand, false-negatives seem to be mostly due to motion artifacts present in the segments, as illustrated in Figure 5, even a small portion of artifacts affects significantly since the distorted portion is spread through several subsegments of the ECM. Therefore, to avoid misclassification a noise reduction block is recommended to attenuate irrelevant information that could confuse the algorithm.

Performance might be improved if more classes are considered while training the CNN. However, the proposed methodology requires balanced datasets for training, and thus a similar number of recordings for each additional class should be available. The latter is not feasible given the limited access to annotated databases for different arrhythmias. The main advantage of the presented approach is that once the $\mathrm{CNN}$ is trained, the detection is made directly on ECG signals containing at least 10 heartbeats which is not possible in other methods that require a buffer of data before making the first AF detection [6]. This could be very useful to analyze signals of short duration such as those resulting from a resting ECG.

\section{Conclusions}

This study presents a new approach for automatic detection of AF episodes as short as 10 beats. For this purpose, segments of ECG were converted to ECM images, and fed to a $\mathrm{CNN}$ for classification. The $\mathrm{CNN}$ was trained using the NSRDB and the LTAFDB, and achieved accuracy of $86.95 \%$ when tested on the AFDB.

\section{Acknowledgements}

This study is framed inside MY-ATRIA consortium and has received funding from the European Union's Horizon 2020 research and innovation programme under the Marie Skłodowska-Curie grant agreement No.766082

\section{References}

[1] Roten L, Schilling M, Häberlin A, Seiler J, Schwick NG, Fuhrer J, Delacrétaz E, Tanner H. Is 7-day event triggered ECG recording equivalent to 7-day Holter ECG recording for atrial fibrillation screening? Heart 2012;98(8):645-649.

[2] Kirchhof P, Benussi S, Kotecha D, Ahlsson A, Atar D, Casadei B, Castella M, Diener HC, Heidbuchel H, Hendriks J, et al. 2016 ESC guidelines for the management of atrial fibrillation developed in collaboration with EACTS. Eur J Cardiothorac Surg 2016;50(5):e1-e88.
[3] Li D, Tian F, Rengifo S, Xu G, Wang MM, Borjigin J. Electrocardiomatrix: A new method for beat-by-beat visualization and inspection of cardiac signals. J Integr Cardiol 2015; 1(5):124-128.

[4] Brown DL, Xu G, Belinky Krzyske AM, Buhay NC, Blaha M, Wang MM, Farrehi P, Borjigin J. Electrocardiomatrix facilitates accurate detection of atrial fibrillation in stroke patients. Stroke 2019;50(7):1676-1681.

[5] Haeberlin A, Roten L, Schilling M, Scarcia F, Niederhauser T, Vogel R, Fuhrer J, Tanner H. Software-based detection of atrial fibrillation in long-term ECGs. Heart rhythm 2014; 11(6):933-938.

[6] Sörnmo L. Atrial fibrillation from an engineering perspective. Springer, 2018.

[7] Xia Y, Wulan N, Wang K, Zhang H. Detecting atrial fibrillation by deep convolutional neural networks. Computers in Biology and Medicine 2018;93:84-92.

[8] He R, Wang K, Zhao N, Liu Y, Yuan Y, Li Q, Zhang H. Automatic detection of atrial fibrillation based on continuous wavelet transform and 2D convolutional neural networks. Frontiers in Physiology 2018;9:1206.

[9] Mitchell R, Michalski J, Carbonell T. An artificial intelligence approach. Springer, 2013.

[10] LeCun Y, Boser BE, Denker JS, Henderson D, Howard RE, Hubbard WE, Jackel LD. Handwritten digit recognition with a back-propagation network. In Advances in Neural Information Processing Systems. 1990; 396-404.

[11] Krizhevsky A, Sutskever I, Hinton GE. Imagenet classification with deep convolutional neural networks. In Advances in Neural Information Processing Systems. 2012; 1097-1105.

[12] Goldberger AL, Amaral LA, Glass L, Hausdorff JM, Ivanov PC, Mark RG, Mietus JE, Moody GB, Peng CK, Stanley HE. Physiobank, physiotoolkit, and physionet: components of a new research resource for complex physiologic signals. Circulation 2000;101(23):e215-e220.

[13] Petrutiu S, Sahakian AV, Swiryn S. Abrupt changes in fibrillatory wave characteristics at the termination of paroxysmal atrial fibrillation in humans. Europace 2007;9(7):466-470.

[14] Moody G. A new method for detecting atrial fibrillation using rr intervals. Computers in Cardiology 1983;227-230.

[15] Goodfellow I, Bengio Y, Courville A. Deep Learning. MIT Press, 2016. http: / /www. deeplearningbook.org.

[16] Ioffe S, Szegedy C. Batch normalization: Accelerating deep network training by reducing internal covariate shift. arXiv preprint arXiv150203167 2015;

[17] The MathWorks I. Deep Learning Toolbox. Natick, Massachusetts, United States, 2019. URL https://www mathworks. com/help/deeplearning/.

Address for correspondence:

Ricardo Salinas-Martínez

Via G. di Vittorio 21b, 40013 Castel Maggiore, Italy

Ricardo.Salinas@hillrom.com 